\title{
3. On Walker's American Types of Lepidoptera in the Oxford University Museum. By W. Schaus, F.Z.S.
}

[Received April 30, 1896.]

The following notes on Walker's American types in the Oxford University Museum are to be followed by others, wherein the complete synonymy will be given of many of the species, and references will also be made to those species which as yet remain unidentified. My special thanks are due to Prof. E. B. Poulton, F.R.S., for the trouble he took in selecting the American Moths from the Hope Collection, and sending them family by family to the British Museum to be compared with the collections there and with my own collection; and to Mr. G. F. Hampson for his untiring kindness and assistance. Those species marked with an asterisk are represented in my own collection; those of which I do not possess specimens, and which are not in the British Museum either, have been figured in duplicate, one set of figures being placed in the Entomological Department of that Museum. The species described by Walker in parts 27-30 of his Catalogue, and in the corresponding portions of the Supplement, as being in "Coll. Saunders" never formed part of the Hope Collection of the Oxford Museum, but have lately been purchased by the British Museum, where the types are now to be found.

The references given refer to Walker's 'List of the Specimens of Lepidopterous Insects in the Collection of the British Museum.'

VoL. III.

P. 775. * Norape puella described from the Fry coll. is now in the Saunders coll., and is the same as Avchylus pectoralis, Walk.

Vol. IV.

P. 801. Sarsina purpurascens. I have not yet been able to find the type.

P. 895. *Darala falcata =Lonomia submacula, Walk.

P. 957. 1 sychagrapha floccosa. Not identified.

Vol. V.

P. 1046. "Naprepa camelinerdes is a distinct species belonging to the Notodontidæ.

P. 1115. Laruma heterogenea is a distinct species, the genus being the same as Hydrias.

P. 1152. Nesara apicalis belongs to the genus Ocha, Walk.

P. 1155. * Pamea excavata and Pamea notata are the sexes of one species.

P. 1155. *Pamea vittata belongs to the genus Carthara, Walk.

P. 1170. Rosema deolis is not Cramer's species, and I therefore propose the name of $R$. walkeri for it ; the species is well described by Walker. 
Vou. V. (continued).

P. 1176. *Tepilia biluna is the same as Phecada confinis, Walk.

P. 1195. "Lonomia albigutta is a well-known species, but will no doubt sink as a synonym of one of Cramer's species.

Vor. VI.

P. 1264. Tropaca dictynna. Evidently a distinct species and not a mere var. of $T$. luna.

P. 1300. *Hyperchiria memusa. A good species allied to H. leucane, Hbn.

P. 1301. *Hyperchivia cinerea. The $q$ of $H$.janeira, Westwood.

P. 1301. Hyperchivia pyrrhomelas is a good species.

P. 1303. *Hyperchiria submacula has been redescribed by Boisduval as $H$. dioxippus.

P. 1313. *Hyperchiria vagans is a species of Dirphia.

P. 1326. Rhescynthis meander. This is well figured by Maassen and Weymer.

P. 1329. *Dysdamonia glaucescens. A distinct species.

P. 1338. Mimallo plana belongs to the genus Perophora.

P. 1364. *Dirphia multicolor is a distinct and well-known species.

P. 1402. *Hydrias albidifascia. A distinct species.

P. 1402. *Hydrias plana. A distinct species.

P. 1403. Hydrias confusa. The type is the only specimen I have seen of this species.

P. 1507. Hydrias tenebrosa. Not identified.

Vou. VII.

P. 1598. Seryda cincta. Not identified.

1. 1601. Pseudomya consolala belongs to the genus Lamocharis.

P. 1602. Pseudomya desperata. Closely allied to P. tipulina, Hübn.

P. 1602. Gymnelia bijuncta. Subsequently described by Walker as $G$. consociata.

P. 1603. Gymnelia simplex. A distinct species.

P. 1603. *Gymnelia xanthocera. A distinct species.

P. 1604. Isanthrene orlyneroides is a species of Erruca.

P. 1606. Preilosoma sperans belongs to the genus Marissa.

P. 1606. Pocilosoma insperata also belongs to the genus Marissa.

P. 1607. Pocilosoma gaudens belongs to the genus Dycladia.

P. 1608. *Lagaria acuminata. Redescribed and figured by Schaus in Lep. Am. as Gartha dalsa.

P. 1609. Lamocharis contracta belongs to the genus Erruca.

P. 1610. Ilipa tenthredoides. Also in B. M.

P. 1611. Ilipa scita=S. hilaris, Walk., in B. M.

P. 1613. Cosmosoma marginatum is a distinct species.

P. 1615. *Eurata transiens $=$ Dycladia lucetius, Cr.

P. 1617. *Eunomia vacillans is a good species.

P. 1617. *Eunomia abdominulis = Corematura chrysogastra, Perty.

P. 1618. Eunomia separuta. Not identified.

P. 1619. Pseudosphea consobrina. Also in B. M.

P. 1619. Pseudosphex cognata=Ps. fasciolata, Butl., in B, M.

P. 1620. Myrmecopsis apaoa $=$ Ps. vespa, H.-S., fig. 426 . 
VoL. VII. (continued).

P. 1627. Calonota perspicua. A distinet species.

P. 1628. Calonota interrupta. Allied to the preceding.

P. 1629. *Pampa acuminata. Redescribed and figured by Schaus in Lep. Am. as Sychesia hartmanni.

P. 1629. Pampa fusiformis=Scepsis trifasciatı, Butl., in B. M.

P. 1634. *Automolis angulosa $=A$. flavicinctus, H.-S.

P. 1635. Automolis saturata. Figured by Felder in the 'Reise d.

Novara,' t. cii. f. 5, as A. protexta.

P. 1635. Automolis basalis. A distinct species.

P. 1636. *Automolis chrysomelas $=$ A. geometrica, Feld.

P. 1636. *Automolis leucomela. Figured in the 'Biologia Centr.Amer.' by Druce as Pterygopterus superba.

P. 1638. *Automolis reduct $a=$ Sutonocrea incertus, H.-S.

P. 1638. Eucerea rhodophila. A distinct species.

P. 1639. *Eucerea latifascia $=E$. archias, Stoll.

P. 1639. Eucerea mitigata $=$ E. reticulata, Butl., in B. M.

P. 1640. *Eucerer albiceps. Gen. nov., allied to Pseudapistosic, Mösch.

P. 1640. *Eucerea discolor. A very distinct species.

P. 1644. Consoprium divisum. Also in B. M.

P. 1645. *Josia mitis. Also in B. M.

P. 1647. * Scea puella. Belongs to the Chrysuregina, and was described by Warren as Siemniomimu albiapicalis.

P. 1647. Scea infans. A good species.

P. 1648. Ephialtias simplex $=E$. abrupta, Hübn.

P. 1649. Leucopsumis guttipalpis. A distinct species.

P. 1650. Corydalla cryptoleuca. A distinct species.

P. 1651. Euagra discalis. A distinct species.

P. 1652. Agyrta secta is a Pyralid=Erilusa dioptalis, Walk.= E. pseudauxo, Feld.

P. 1652. Agyrta nana. A distinct species.

P. 1654. Pericopis larvata. A good species.

P. 1655. Pericopis disjuncta. Not identified.

P. 1656. Lyces plagiferc. A distinct species.

P. 1657. Lyces albiventris belongs to the genus Flavinia, Walk.

P. 1657. Flavinia fusifera. Also in B. M.

P. 1659. Avela diversa. Not identified.

P. 1661. *Caralisa editha. Genus allied to Charidea.

P. 1661. "Sangula beata. A well-known species.

P. 1662. Melanchroia subvittat $a=R$ Rosus posticus, Walk.

P. 1663. Arina obtusa. A distinct species.

P. 1677. Phaochlaena quadricolor. A distinct species.

P. 1678. *Spiris nigrilinea. A distinct species.

P. 1679. * Pagara venosa = Agoraea longicornis, H.-S.

P. 1693. *Daritis marginalis=Pericopis lycaste, Klug.

P. 1698. * Isia intricata. Genus allied to Ecpantheria.

P. 1701. *Pithea ferruginea. Genus allied to Phagoptera.

P. 1702. Aloa lanceolata. Gen. nov., allied to Halisidota.

P. 1704. Amastus mesorhoda. A distinct species. 
VoL. VII. (continued).

P. 1704. *Opharus basalis. A distinct species.

P. 1705. Halesidota flavescens. Genus allied to Idalus.

P. 1706. *Halesidota apicalis. A good species.

P. 1706. Halesidota discalis. Not identified.

P. 1706. Halesidota catenata=Phagoptera flavopunctata, Walk.

P. 1707. Halesidota corticea. Not identified.

P. 1707. *Halesidota semivaria. Gen. nov.

P. 1707. *Halesidota basipennis=Phagoptera subtilis, Butl.= Sychesia fimbria, Mösch.

P. 1708. Halesidota semirufa. A good species.

P. 1708. *Halesidota antica. Belongs to the genus Euchates.

P. 1709. *Halesidota niveigutta. A distinct species.

P. 1709. *Halesidota binotata, gen. nov. This species was described by Boisduval as Phag. cinnamomea.

P. 1709. *Halesidota disciplaga. Redescribed by Walker as $H$. breviuscula.

P. 1710.*Halesidota cingulata. Belongs to the genus Mazaras.

P. 1710. *Ambryllis neurophylla. Redescribed and figured by Dognin as Ecpantheria hebona.

P. 1712. *Minara pardalina =Drymonia histrionica, H.-S.

P. 1714. *Podalia vesta $=$ Megalopyge or silochus, Cr.

P. 1726. Eloria mosta. A distinct species.

P. 1727. Eloria lucida. Also in B. M.

P. 1728. *Simena luctifera. A well-known Geometrid.

P. 1737. *Colabata lineosa=Andriasa marginalis, Walk.

P. 1749. Notodonta plagiata. Not identitied.

P. 1761. Candyba punctata. A distinct species.

VoL. VIII.

P. 47. AEgeria buprestiformis. A good species.

P. 55. Aigeria panisciformis. A good species.

P. 55. AÉgeria blaciformis = Sesia aequatus, Walk., in B. M.

P. 115. Enys prominens. Figured by Felder in the 'Reise d. Novara,' t. Ixxxii. f. 5 , as T'. smerinthoides.

P. 190. *Pachylia inconspicua $=P$. resumens, Walk.

P. 197. *Oryba robusta $=0$. achemenides, Cr.

P. 233. *Laparc bombycoides = Ellema harrisii, Clem.

VoL. IX.

\section{Noctuid $x$.}

P. 61. *Acronycta declarat $\iota=$ Mamestra adjuncta, Bdv.

P. 195. *Prodenia latifascia = Prodenia variolosa, Wlk.

P. 235. *Mamestra crist $a=M$. obscurus, Dogn. $=M$. infernatis, Schs.

VoL. X.

P. 268. * Celana plagiata belongs to the genus Perigea.

P. 269. *elana expuncta belongs to the genus Oligitı.

P. 401. *Graphiphor inaja= Noctuce major, Gn.

P. 430. *Taniocampic subsocic. A distinct species. 
Vol. XI.

P. 588. Hadena subjecta. A distinct species.

P. 606. Casandria emittens. Not identified.

P. 629. *Xylina bipunctata. A good species.

P. 737. Agrotis emittens belongs to the genus Acanthodica, Schs.

P. 739. Agrotis hirtipalpis. A distinct species.

Vor. XII.

P. 787. Acontia decisa. Not identified.

P. 819. *Xanthoptera aurifera. A distinct species.

P. 819. *Xanthoptera furcata. A distinct species.

P. 848. *Palindia punctangulata $=$ P. alabastrariı, Hübn.

P. 917. *Plusia admonens $=P$. basigera, Walk.

P. 977. Lymphorta unilinea. Not identified.

Vor. XIII.

P. 1007. Scadisa designans. Not identified.

P. 1034. *habuata ampla. A good genus and species.

P. 1058. *Homoptera configurata. A distinct species.

P. 1094. * Canipeta illustrans $=C$. suttea, $\mathrm{Gn}$.

P. 1094. Ccenipeta abscondens. Not identified.

P. 1132. Stictoptera subaurata. Also in B. M.

P. 1171. Coronta surrepens $=$ Melipotis strigifera, Walk.

P. 1231. Graphigona antica. Not identified.

Vol. XIV.

P. 1251. Brujas basicinct $a=$ Ramphia evinga, Gn.

P. 1255. *Brujas laticincta $=$ Letis magna, Gmel.

P. 1271. *Letis implens = Letis cortex + , Gn.

P. 1273. *Letis integra $=$ Letis cortex ${ }^{\circ}, \mathrm{Gn}$. The type of $L$. integra is now in the B. M.

P. 1274. *Letis albicans. The type of this species is also in the B. M. ; it is in very poor condition, but represents, I believe, a very distinct species, of which 1 possess several perfect specimens.

$\mathrm{P}:$ 1284. *Latebraria quadriplaga $=R$. evinga ơ, $\mathrm{Gn}$.

P. 1323. Hypopyra configurans. Not identified.

P. 1472. * Poaphila revoluta = Hypena scabra, Fabr.

Vol. XV.

P. 1555. Orthogramma perseverans. A distinct species.

P. 1568. * Thermesia signans $=$ Azeta uncas, Gn.

P. 1569. Thermesia tripunctifer $a=0$. perseverans, Walk.

P. 1572. *Thermesia lenis = Epidromia pannosa, Gn.

P. 1584. Selenis amans. Not identified.

P. 1620. Hypernaria integrans $=H$. augusta, Cr.

P. 1620. Hypernaria interponens $=H$. augusta, Cr.

P. 1629. *Plaxia falcigera. A distinct species.

P. 1632. Palyna metagona = Galapha sublineata, Walk.

P. 1640. Edyma significans. Genus allied to Thermesia.

P. 1642. Pessida interlineata. Not identified.

P. 1644. *Ausava triplaga. A good genus and species. 
VoL. XV. (continued).

P. 1646. * Cymatophora temperans=Orthodes infirma, $\mathrm{Gn}$.

P. 1647. *Bryophila expetita. A distinct species.

P. 1653. Molynela humeralis=Oroatis signata, Butl., in B. M.

P. 1657. Eragisa lanifera. A Notodont allied to the genus Elymiotis.

P. 1659. *Leucania cinereicollis. A distinct species.

P. 1663. Alpesa villicosta. Also in B. M.

P. 1669. *Egabra certissima= Azelina hadularia, Gn., a Geometer.

P. 1669. * Bacula chromatophila $=$ Dyops ocellata, Cr.

P. 1677. *Laphygma congress $\iota=$ Dantona discerpta, Walk.

P. 1677. *Laphygma trientiplaga $=$ Oligia expuncta, Walk.

P. 1685. *Mamestra dentistrigata belongs to the genus Perigea, Gn.

P. 1687. Celana inquieta. A distinct species.

P. 1690. Celaena tetera. A distinct species.

P. 1690. *Celana subobliqua. A distinct species.

P. 1694. Caradrina distans $=$ Celana inquieta, Walk.

P. 1694. * Caradrina laphygmoides =Perigea concisa, Walk.

P. 1699. *Agrotis interferens. A distinct species.

P. 1711. *Cirradia satellifera $=$ Anomis exaggerata, Gn.

P. 1714. *Dianthocia eros. Also in B. M.

P. 1718. *Valeria angulipluga. A distinct species.

P. 1719. *Angitia directa. A distinct species.

P. 1725. *Hadena vacillans $=$ Perigea fidelia, Druce, figured in 'Biol. Centr.-Amer.'

P. 1726. *Hadena pennitarsis $=H$. tessellata, Sepp.

P. 1730. *Acroria villipes = Dargida niphanda, Druce, figured in 'Biol. Centr.-Amer.'

P. 1731. Lithomia buddha. A distinct species.

P. 1733. *Xylina patefacta. A good species.

P. 1742. *Nasia moesta. A distinct species.

P. 1742. *Nystalea aquipars $=N$. seminivea, Walk.

P. 1754. Pastona rudis. A good genus and species.

P. 1767. Palindia spectabilis $=P$. perlata, Gn.

P. 1768. Palindia caruleilinea. Also in B. M.

P. 1773. *Agraga fimbripes. A good genus and species.

P. 1774. *Penicillaria abscondens. Well figured in the 'Biologia Centr.-Amer.'

P. 1782. *Abrostola interrumpens $=$ Edema nana, Walk.

P. 1784. *Adranc pseudopsis=Ingura fuscescens, Walk.

P. 1785. * Plusia intract $a=P$. basigera, Walk.

P. 1788. Thyria aurifundens. A distinct species.

P. 1790. *Gonodonta ditissina. A good species.

P. 1793. * Cosmophila punctifera=Gonitis editrix, Gn.

P. 1796. * Selambina trajiciens. Also in B. M.

P. 1799. *Homoptera separabilis belongs to the genus Campometra.

P. 1799. *Homoptera hadenoides belongs to the genus Nania.

P. 1800. *Homoptera stipatura belongs to the genus Phosplila.

P. 1800. Homoptera indecidens belongs to the genus Homopyralis

P. 1814. Stibcerc hostilis. Not identified. 
Vol. XV. (continued).

P. 1819. Letis discopalina. New genus near Homoptera.

P. 1844. *Phurys asseverans. A good species.

P. 1845. *Onoba trogonoides=Baniana pohli, Feld.

P. 1846. *Phuphena fusipennis = Perigea ? trilinea, Schs.

P. 1851. Escua extollens. Belongs to the I'hermesiida.

P. 1852. Carvania clandestina. A Deltoid.

$\mathrm{P}$ 1856. Amphigonia postponens. Also in B. M.

P. 1856. *Thermesia imitatura belongs to the genus Physula.

P. 1857. Thermesia bigutta. A distinct species.

P. 1857. Obucola expandens belongs to the genus Peosina, Gn.

P. 1858. Capnodes extima. A distinct species.

P. 1859. Donacesa miricornis. Allied to Orthogramma, Gn.

P. 1860. *Hypernaria discessura. A distinct species.

Vor. XVI.

Delioidex and Prialida.

P. 80. *Hypena acclinalis $=$ Hypena anicina, Druce, Biol. Centr.Amer., Het. i. p. 434 (tab. xxxv. fig. 13).

P. 81. *Hypena mostalis belongs to the genus Rejectaria, Gn., and is allied to $R$. cocytalis, Gn.

P. 81. *Hypena hastatalis. Subsequently described by Walker as Crymona receptalis, Trans. Int. Soc. Lond. 3rd ser. i. p. 117. The species will stand as Crymona hastatalis.

P. 186. *Bocana orionalis = Simplicia tibialis, Felder, 'Reise d. Novara,' t. cxx. fig. 43.

P. 190. Ipnea erebusalis belongs to the genus Ceromacra, Gn., of the Focillina.

P. 242. *Bleptina proliferalis. A distinct species.

P. 251. Galanda helrusalis. A good species belonging to the Hypenidæ.

VoL. XVII.

P. 438. *Cataclysta? pegasalis belongs to the genus Dicymolomia, and was redescribed by Walker as C.principalis, Cat. Het. B. M. vol. xxxiv. p. 1333.

P. 442. Cataclysta pantheralis. A good species.

P. 443. Cataclysta phadralis belongs to the genus Ambia.

VoL. XVIII.

P. 513. *Prakellura dardusalis=Mesocondyla stigmatalis, Led.

Wiener ent. Monatsch. vol. vii. t. 13. fig. 3.

P. 513. *Phakellura eumeusalis belongs to the genus Sestia.

P. 522. Margaronia argealis $=M$. aquarialis, Led. MS., in B. M.

P. 523. Margaronia ianthealis belongs to the genus Sozoa, Walk., and was redescribed by Walker as Sozoa costalis, Cat. Lep. Het. B. M. vol. xxxiv. p. 1373.

P. 523. *Margaronia hypheusalis. A good species.

P. 536. Chromodes armeniacalis. A good species.

P. 593. Botys mnemusalis. Not identified. 
VoL. XVIII. (continued).

P. 595. Botys eubulealis belongs to the genus Hyalea.

P. 595. *Botys nerissalis belongs to the genus Phlyctania, and is allied to $P$. cuneifera, Warr., in B. M.

P. 596. * Botys cypraalis=Tanaura sublutalis, Warr., in B. M.

P. 597. *Botys remusalis = Anarmodia inscriptalis, Gn.

P. 600. Botys pyrrhusalis. Not identified.

P. 601. *Botys chlorisalis belongs to the genus Pantographa, Led.

P. 602. *Botys melitealis = Blepharomastix colubralis, Gn.

P. 609. * Botys quirinalis = Sylepta pactolalis, Gn.

P. 610. Botys thalloalis=Allactostigma inguinalis, Gn.

P. 610. "Botys peranthusalis = Eulepte concordalis, Gn., in B. M.

P. 611. Botys niavialis. Not identified.

P. 615. Botys elathealis belongs to the genus Coelorhyncidia, Hamps., Hydrocampina.

P. 615. * Botys sylvialis = Hyalorista myopicalis, Led.

P. 617. *Botys agenoralis = Microthyris prolongalis, $\mathrm{Gn}$.

P. 617. Botys damonalis belongs to the genus Sylepta.

P. 618. Botys pandaralis belongs to the genus Colorhyncidia.

P. 622. *Botys evippeulis belongs to the genus Syllepis.

P. 623. Botys persiusalis=Phostria confluentalis, Warr., in B. M.

P. 626. Botys asiusalis=romalis, Druce, belongs to the genus Massepha, Walk.

P. 627. *Botys odiusalis belongs to the genus Sylepta.

P. 627. *Botys pieralis =Azochis gripusalis, Walk. vol. xviii. p. 542 .

P. 628. *Botys turnusalis = Pranesta scyllatis, Walk. vol. xviii. p. 565.

P. 629. * Botys claudiusalis belongs to the genus Calamachrous.

P. 631. *Botys sabulis $=$ Hedylepta vulgalis, $\mathrm{Gn}$.

Vol. XIX.

P. 833. Geroda xeneusalis. A Noctuid belonging to the Trifince near the genus Amyna.

P. 835. *Nachaba congrualis. A distinct species of the Chrysaugina.

P. 835. Nachaba oppositclis. A distinct species of the Chrysaugina.

P. 836. *Licha undilinealis = Leucania cicatrix, Feld. Reise d. Nov. t. cix. fig. 8 .

P. 837. Lancia phrontisalis. Not identified.

P. 838. Pterhemia otusalis $=P$. uncinalis, Hübn.

P. 839. *Lascoria phormisalis belongs to the genus Tortricodes, and is figured in Biol. Centr.-Amer., Het. i. t. 38. figs. 14,15 .

P. 840. *Hypena deldonalis. Very similar to Britha hirtipalpis, Walk.

P. 841. *Hypena xenaresalis. A distinct species.

P. 842. Hypena lyrcusalis. A distinct species.

P. 842. *Hypena iysoalis $=H$. exoletalis, Gn. 
VoL. XIX. (continued).

P. 843. *Hypena degesalis. A distinct species.

P. 844. *Hypena subbasalis. A distinet species.

P. 846. * Mursa calisalis. A distinct species.

P. 846. Gisira endeisalis. Not identified.

P. 847. Hormisa abeluxalis = Pluurys basilans, Gn.

P. 850. Langasa perillalis. A good genus and species.

P. 851. *Hydara biconalis = Psaliodes paleata, Gn., a Geometrid.

P. 857. *Herminia moeonalis. A very distinct species belonging to a new genus.

P. 857. *Herminia apisalis belongs to the genus Palthis.

P. 858. * Herminia asteralis $=$ Palthis aspisalis, Walk.

P. 859. Hermina baresalis=Mastygophorus epitusalis, . Walk. vol. xvi. p. 150.

P. 862. *Bleptina metopealis =B. confusalis, Gn.

P. S63. Bleptina pollesalis. Apparently a faded specimen of B. confusulis, Gn.

P. 863. *Bleptina bogusalis belongs to the genus Heterogramma, Gn.

P. 864. *Bleptina opiteralis $=$ Tortricodes phormisalis, Walk.

P. 864. *Bleptina oppialis = Heterogramma enclorealis, Gn.

P. 865. "Bleptina bizialis = Palthis agroteralis, Gn.

P. 866. Bleptina styrusalis. A distinct species.

P. 866. *Bleptina capioalis = Het. bogusalis, Walk.

P. 867. *Bleptina fadusalis=also Het. bogusalis, Walk.

P. 868. Bleptina ophelasalis=B. styrusalis, Walk. p. 866 .

P. 868. *Bleptina ceusalis = Het. bogusalis. Walk.

P. 869. Bleptina abarusalis. Not a Bleptina. Near the genus Catada.

P. 869. Bleptina stalemusalis. A distinct species.

P. 870. *Bleptina pagasusalis. A distinct species.

P. 870. *Bleptina bogesalis = B. confusalis, Gn.

P. 871. Bleptina aeatusalis. A good species.

P. 880. *Renia ? decelusalis = Bleptina pagasusalis, Walk.

P. 881. Renia? lytocalis. A distinct species.

P. 881. *Renia ? borgesalis belongs to the genus Megachyta.

P. 885. Megatomis euphrionalis. A good species.

P. 893. *Pyralis antenoralis belongs to the genus Bradina.

P. 893. Pyralis thiastoralis belongs to the genus Rhodoneura.

P. 894. Pyralis ninniusalis belongs to the genus Hypolamprus of fam. Thyrididæ.

P. 894. Pyralis? byzesalis. Not identified.

P. 896. *Pyralis? atialis belongs to the Tineidæ, genus Tortricomorpha $=$ varipes and vescatilis, Walk.

P. 923. *Rhodaria probalis = Hamatia panopealis, Walk.

P. 927. Desmia pisusalis=reconditalis and minualis, Walk., belongs to the genus Diathrausta.

P. 928. *Desmia bulisalis $=$ D. ufeus, Cr.

P. 928. Desmia nerinalis belongs to the genus Diathrausta.

P. 936. "Samea calonalis belongs to the genus Sciorista. 
VoL. XIX. (continued).

P. 937. * Samea luccusalis =S. ecclesialis, Gn.

P. 944. *Hymenia ? phrasiusalis $=H$. perspectalis, Hübn.

P. 945. *Isopteryx ? staiusalis $=$ Phlyctonia scitalis, Led.

P. 948. Lineodes peridialis. Not identified.

P. 949. *Hydrophysa adiantialis. A good species.

P. 949. Hydrophysa capiosalis belongs to the genus Parthenodes, Gn.

P. 950. Oligostigma scuthesalis belongs to the genus Aulacodes, and is the same as $A$. psyllalis, Gn.

P. 951. *Oligostigma iasusalis. A distinct species.

P. 952. Cataclysta aglesalis. A distinct species.

P. 952. * Cataclysta casoalis. A distinct species.

P. 953. * Cataclysta aealis. A distinct species.

P. 954. *Cymoriza atalis. Nymphula lotialis, Feld., 'Reise d. Novara,' t. cxxxv. tig. 4 .

P. 955. Cymoriza bocusalis. A good species.

P. 955. Cymoriza badiusalis belongs to the genus Nymphula.

P. 956. * Cymoriza narcissusalis belongs to the genus Musotima.

P. 956. Cymoriza abrotalis. A distinct species.

P. 957. Cymoriza bolusalis = C. damescalis, Gn., in B. M.

P. 965. Zebronia lacrinesalis belongs to the genus Conchylodes.

P. 966. *Zebronia deicoonalis belongs to the genus Aripana.

P. 966. Zebronia caliusalis. Not identified.

P. 967. *Zebronia bunusalis = Aripana levinia, $\mathrm{Cr}$.

P. 968. Zebronia? bialis. Not identified.

P. 969. Zebronia ? abronalis=Platytes pusillalis, Hübn.

P. 984. *Botys tytiusalis belongs to the geuns Isosalbia.

P. 985. "Botys autoclesalis belongs to the genus Notaspis, Led.

P. 985. * Botys azionalis = Dolichosticha cochrusalis, Walk. p. 959.

P. 986. *Botys dimichealis belongs to the genus Tegostoma.

P. 986. *Botys graviusalis = Samea paotinalis, Warr., in B. M.

P. 987. Botys imbrexalis belongs to the genus Ceratoclasis, Led.

P. 987. *Botys nocmonalis $=$ Phlyctania nerissalis, Walk. vol. xviii. p. 595 .

P. 988. Botys acilialis. Not identified.

P. 988. * Botys gelliasalis = Steniodes lutealis, Snell., in B. M.

P. 1000. *Ebulia? ialis belongs to the genus Hapalia.

P. 1011. *Pionea camaroalis belongs to the genus Tholeria.

P. 1012. Pionea ademonalis. A distinct species.

P. 1019. *Illice batialis. A Lithosid ; also in B. M.

P. 1020. Davara azonaxsalis. A Phycyta.

P. 1021. *Dantona busalis $=N$. discerpta, Walk. This is a Noctuid, and will stand as Dantona discerpta, Walk.

P. 1022. * Motya abseusalis. A good species.

\section{Geometride.}

Vol. XX.

P. 23. *Chorodes invariaria $=$ Asschropteryx tetragonata, $\mathrm{Gn}$.

P. 45. *lysia absconditaria $=$ Rio form of $C$. tasima, Cr. 
VoL. XX. (continued).

P. 52. * Oxydia noctuitaria $=0$. vitiligata, Feld.

P. 61. Chorodes assyllusaria. Not identified.

P. 62. *Mucronodes oricusaria = Oxydia trychiata, Gn.

P. 64. "Oxydia alpiscaria = Oxydia apidania, Cr.

P. 64. Oxydia cariaria. A distinct species.

P. 79. *Drepanodes agronaria. A distinct species.

P. 94. * Gynopteryx telysaria $=G$. rhombaria +, Gn.

P. 109. *Apicia rhumata =A. lintearia, Gn.

P. 109. Apicia nazadaria. A distinct species.

P. 110. *Apicia uxiaria = Gynopteryx seriaria o , Gn.

P. 110. *Apicia alphiusaria. A distinct species.

P. 111. *Apicia ? gaumaria = Diepanodes hamulata, Gn.

P. 147. *Nematocampa varicata. A good species.

P. 171. * Caberodes carcearia $=C$. fundaria, Gn.

P. 194. * Azelina asilasaria $=A$. denticulata, Butl.

P. 195. Azelina amyclaric. Not identified.

P. 220. * Urapteryx palindiaria $=$ Aschropteryx onustaria, Hübn.

P. 222. *Choerodes contemnaria = Certima muscistriyata, Gn.

P. 223. Oxydia nerisaria. A distinct species.

P. 225. * Drepanodes arnataria $=D$. pholata, Gn.

P. 225. *Drepanodes excavaria. A distinct species.

P. 226. *Drepunodes apertaria = Pyrinia erythroceplalata, Gn.

P. 226. *Drepanodes grata. A distinct species.

P. 227. *Drepanodes diffundaria. A distinct species.

P. 229. Pyrinia madiaria. A distinct species.

P. 229. * Pyrinia mephasaria. A distinct species.

P. 230. *Pyrinia alcandraria. A distinct species.

P. 234. *Apicia apaliusaria $=$ A. spinetaria o", Gn.

P. 235. *Apicia significaria= Renodes brevipalpis, Gn.

P. 236. Apicia incrassata. Not identified.

P. 236. *Apicia extimaria. A Boarmid of the gen. Ectropis, Hiibn.

P. 237. *Apicia atillaria =Renodes brevipalpis, $\mathrm{G}_{\mathrm{n}}$.

P. 238. Apicia molusaria. Not identified.

P. 238. *Apicia ansercria belongs to the genus Drepanodes.

P. 239. *Apicia inficitaria = Apicia asopia, Druce.

P. 240. Epione allutiusaria. Not identified.

P. 240. Epione ardysaria. Not identified.

P. 241. *Epione mitranaria. A distinct species.

P. 241. *Epione relictaria. A distinct species.

P. 242. *Angerona expulscria = Alana transitaria, Gn.

P. 246. *Nematocampa nyparia belongs to the genus Anictes, Warr.

P. 251. *aberodes asanderaria. A distinct species.

P. 245. Dasmeuda alcimusata. A distinct species.

P. 254. *Eusenea ceniasaria. In B. M. under the genus Azelina.

P. 256. *Azelina fusaria =A. stolidata, Gn.

P. 262. *Halesa cenetusaria $=H$. glauca, Butl.

P. 268. *Laudosia buddloraria = Cirsodes acuminata, Gn.

P. 269. *Bassania amethystata. A good species.

P. 275. Berambe gallaric. Not identified. 
Vou. XXI.

P. 296. *Meticulodes xylochromaria $=M$. xylinaria, Gn.

P. 354. Boarmia contraria belongs to the genus Euctenectropis.

P. 354. *Boarmia vacillaria $=$ B. vacillaria, $\mathrm{Gn}$.

P. 357. Boarmia mollearia. A distinct species.

P. 357. *Boarmia detractaria $=T$ Tephrosia dimidiaria, Gn.

P. 358. *Boarmia guttularia=Bryoptera leprosata, Gn.

P. 359. Boarmia defimaria belongs to the genus Euctenectropis.

P. 360. *Boarmia delinquaria. A distinct species.

P. 361. *Boarmia larentiata $=$ Pterocypha umbrinata, Gn.

P. 362. *Boarmia denticularia $=$ Tephrosia hyberniaria, Gn.

P. 363. Boarmia consimilaria. A distinct species.

P. 363. Boarmia locupletaria belongs to the genus Pterocypha.

P. 363. Boarmia stigmaria. Not identified.

P. 411. *Tephrosia incongruaria =Bryoptera leprosata, Gn.

P. 412. *Tephrosia ineffectaria $=T$ Tephrosia responsaria, Walk.

P. 424. *Paraphia macariata= Semiothisa pernicata, Gn.

P. 425. Paraphia epioneata. A distinct species.

P. 482. Gazena divulsa. A distinct species.

Vol. XXII.

P. 503. *Achlora veniliata = Cambogia insignata, Walk.

P. 503. *Achlora catenularia. A distinct species.

P. 512. Geometra basiplaga. A distinct species.

P. 583. *Racheospila marginiplag $a=R$. ocellata, $\mathrm{Cr}$.

P. 583. *Racheospila satisfacta. A distinct species.

P. 593. Thalera distracta. A distinct species.

P. 662. Hyria subtectata. Not identified.

P. 732. *Acidalia obliviaria. A distinct species.

P. 742. Acidalia justata. Not identified.

VoL. XXIII.

P. 788. *Acirlalia indecretaria $=$ A. obliviaria, Walk.

P. 788. *Acidalia résponsaria. A distinct species.

P. 789. *Acidalia suffusari $=$ Certima muscistrigata, Gn.

P. 799. Timandra viridiplaga. A distinct species.

P. 827. *Ratiaria argentilinen $=$ Urapteryx platinata, Gu.

P. 828. Ratiaria metaxantha. Not identified.

P. 839. *Erosiu leucospilaria. A distinct species.

P. 839. *Erosia semilaria belongs to the genus Schidax.

P. 841. *Erosia lacerataria. A distinct species.

P. 844. Erosia distincta belongs to the genus Psaliodes.

P. 845. *Erosia subsignaria. A distinct species.

P. 859. *Paloda dentifera $=$ Erosia acutangularia, Walk.

P. 910. *Macaria subitaria $=$ Semiothisa triplicaria, H.-S.

P. 911. Macaria mandata. A distinct species.

P. 912. * Macaria concisaria. A distinct species.

P. 913. *Macaria percisaria = Semiothisa gambaria, Huibn.

P. 915. *Macaria continuaria = Semiothisa pernicata, Gn.

P. 919. *Macaria externaria $=$ S. pernicata, Gn.

P. 919. *Macaria fractaria $=$ S. nervata, Gn. 
Vol. XXIV.

P. 1069. Aspilates perlineata. A distinct species.

P. 1081. Alydda lignosata. Not identified.

P. 1100. Gustiana subflexata is a Deltoid.

P. 1264. Thera perarcuata. A distinct species.

Vor. XXV.

P. 1310. * Coremia lateraria $=$ C. fringillata, Gn. ·

P. 1338. *Phibalapteryx mediata=Plemyria fluviata ,, Hiibn.

P. 1339. *Phibalaptery $x$ intrusata $=P$. fluviata ơ, Hübn.

P. 1351. Scotosia nitidulata= Gazena divulsa, Walk.

P. 1369. *Pterocypha divulsata $=$ P. floccosaria , Walk.

P. 1370. *Pterocypha celerata. A distinct species.

P. 1396. * Cidaria perspicuata $=$ C. emberizata, Gn.

P. 1396. *Cidaria patulata. A distinct species.

P. 1397. Cidaria eductata $=$ C. emberizata, Gn.

P. 1397. * Cidaria intercalata. A distinct species.

VoL. XXVI.

P. 1486. Lagyra? dentilineata. Not identified.

P. 1490. Pyrinia reflectaria. A distinct species.

P. 1491. Pyrinia concisata. A distinct species.

P. 1492. Pyrinia subaurata. A distinct species.

P. 1493. Pyrinia saturata. A distinct species.

P. 1493. Pyrinia radiolata. Not identified.

P. 1499. *Rumia defixata $=S$. deprivata, Gn.

P. 1502. Hyperetis tessellata. A distinct species.

P. 1502. *Hyperetis quadrilineata $=$ Semiothisa contorta, Druce.

P. 1505. Endropia? subapicata. Not identified.

P. 1515. Tetracio incequaria. An Apicia allied to cayennaria, Gn.

P. 1533. *Boarmia perspectata $=B$. vacillaria, Gn.

P. 1534. *Boarmia subapicata $=B$. syrniaria, Gn.

P. 1534. Boarmia tenerata is the of $B$. mollearia, Walk.

P. 1553. Caripraa mendaciaria. Not identified.

P. 1560. *Thalassodes glauculata. A distinct species.

P. 1578. *Anisodes congruaria. A distinct species.

P. 1579. Anisodes fimbripedata. Not identified.

P. 1579. *Anisodes ordinata $=$ directata $=$ A. urcearia, Gn. ?

P. 1587. Hyria gavisata. A distinct species.

P. 1587. Cambogia albopunctata. A distinct species.

P. 1599. Acidalia calidata. Not identified.

P. 1600. *Acidalia tepidata. A distinct species.

P. 1600. Acidalia indignaria = Cambogia marcearia, Gn., in B. M.

P. 1626. Erosia integrata. A distinct species.

P. 1627. Erosia subtruncata. Not identified.

P. 1627. *Erosia quadruncata $=$ Phyllodonta caninata, Gn.

P. 1628. *Erosia subpatulata $=$ Epiplema incolorata, Gn.

P. 1629. Erosia bipartaria. Not identified.

P. 1633. Carmala flexata. Not identitied.

P. 1636. *Arcobara microniata= Pigia tergeminaria, H.-S. 
Vol. XXVI. (continued).

P. 1643. Macaria vitriferaria. A distinct species.

P. 1657. Tephrina signataria. A distinct species.

P. 1658. *Tephrina responsaria. A distinct species.

P. 1659. *Tephrina albipunctata. A distinct species.

P. 1687. Cadyanda lugens=Calospila posthumaria, H.-S.

P. 1690. *Budara conversata. A distinct species.

P. 1702. Larentia inquinata=Hammaptera perturbata, Wlk., o .

P. 1710. *Lobophora bifiliferata belongs to the genus Nola.

P. 1718. Phibalapteryx erosiata. Not identified.

P. 1723. *Scotosia spilotata $=$ Homopyralis inderidens, Walk.

P. 1730. Cidaria effrenata. Not identified.

P. 1737. Eubolia vicaria. Not identified.

P. 1754. *Cambogia porphyrinata. A distinct species.

P. 1755. Cambogia turbata. A distinct species.

P. 1755. Acidalia illiturata. Not identified.

P. 1756. Acidalia isographata. A distinct species.

P. 1757. *Erosia niveinotata belongs to genus Dagassa.

P. 1759. Stegania quadrinotata. A distinct species.

\section{Supplement.}

VoL. XXXI.

P. 21. Cotana mediana. A distinct species and good genus.

P. 142. Gerra hyelosioides= Gonora heliconiata, Walk.

P. 257. *Aucula josioides belongs to the Agaristidæ.

P. 304. Elysius signatus. A distinct species.

P. 317. *Addua inclusa $=$ Pericopsis parnassioides, Walk.

Vou. XXXII.

P. 339. *Eloria canescens belongs to the genus Hyalospila.

P. 382. *Compsa saturata $=$ Tarchon trilunula, H.-S.

P. 411. *Notodonta scitipennis $=N$. stragula, Grote.

P. 439. *Parathyris ennomoides belongs to the genus Olcecloslera.

P. 441. *Gopha mixtipennis. A distinct species.

P. 442. *Blera cerruroides. A good species.

P. 442. * Rifargia xylinvides. A good species.

P. 442. *Nagidusa xylocampoides. A good species.

P. 442. Luca herbida. Allied to Heterocampa.

P. 442. *Phastia basalis. A good species.

P. 487. Limacodes concolor. Not identified.

P. 491. Athrula saturnioides $==$ Rolepa delineata, Walk.

P. 516. Siculodes annuligera. A distinct species.

P. 517. Vadata macropterana. A distinct species; the species figured under this name in the 'Biologia Centr.-Amer.' is a new species.

P. 545. *Sosxetra grata. A Noctuid.

P. 579. *Cistissa expansa $=$ Megalopyge fuscescens, Walk.

P. 579. *Batatara fusifascia = Hylesia approximans, Walk.

P. 681. Perigea adornata. Not identified. 
VoL. XXXIII.

P. 731. *Hadena subapicalis $=$ Heterochroma eriopioides, Gn.

P. 780. Azamora basiplaga $=$ Az. tortriciformis, Walk.

P. 798. *Xanthoptera alboflava. A distinct species.

P. 811. *Callopistria vittata=Oligia subobliqua, Walk.

P. 814. * Penicillaria areusa belongs to the genus Ingura.

P. 852. ${ }^{*}$ Hemiceras illucens $=H$. barina, Gn.

P. 861. *Gonitis revers $a=G$. exaggerata, Gn.

P. 866. Coruncala latipennis. Not identified.

P. 877. Homoptera discalis=Platydia mollealis, Walk.

P. 877. Homoptera gratiosa. Not identified.

P. 878. *Homoptera punctilinea. A distinct species.

P. 883. *Homoptera privata $=H$. exhausta, Gn.

P. 883. Homoptera phoceleuca. Not identified.

P. 884. *Homoptera parvula. A good species.

P. 915. Carthara albicosta. Not identified.

P. 998. *Phurys partita belongs to the genus Capnodes.

P. 1026. Thyridospila compta. Not identified.

P. 1027. *Thyridospila quadriocellata. New genus.

P. 1028. Thyridospila amœen $=$ Mulelocha calligramma, Hübn.

P. 1037. *'Thermesia absumens = Euthermesia incxacta, Walk.

P. 1048. *Thermesia caliginosa = Mulelocha exsiccata, Walk.

P. 1049. Thermesia brevistriga. Not identified.

P. 1051. Thermesia divulgata. Not identified.

P. 1052. Thermesia subfixa belongs to the genus Renodes, Gn.

P. 1053. Thermesia conficita. Not identified.

P. 1053. Thermesia inficita. Not identified.

P. 1068. Selenis stipata $=$ S. humeralis, Walk.

P. 1073. Capnodes basalis=Bleptina proliferalis, Walk.

P. 1073. Capnodes schizospila. A distinct species.

P. 1074. *Caprodes concinnula. A distinct species.

P. 1075. Capnodes mundicola is near the genus Dagassa.

P. 1079. Ctypansa obtusa. Not identified.

P. 1081. Hypernaria rudis. Not identified.

P. 1087. *Hypernaria chlorospila $=H$. discessura, Wlk.

P. 1088. *Hypernaria leucospila =Thermesia gemmatalis, Hübn.

P. 1091. Hypernaria punctulosa. Not identified.

P. 1100 . Elixoia subocellata. Not identified.

Vor. XXXIV.

P. 1129. Gaalic dispunctalis= Gustiana sulffexata, Walk.

P. 1132. "Hypena bisignalis. A distinct species.

P. 1132. *Hypena pyralalis $=$ Psatiodes paleata, Gn.

P. 1134. *Hypena disseptalis $=H$. ancara, Druce.

P. 1135. *Hypena dissutalis $=H$. scabra, Fabr.

P. 1145. *Boana semialba. A distinet species.

P. 1158. *Herminia inostentalis $=$ Megachyta borgesalis, Walk.

P. 1158. Herminia figuralis=Gustiana subflexata, Walk.

P. 1160. *Bleptina responsalis $=$ Palthis aspisalis, Walk.

P. 1160. "Bleptina penioillalis belongs to the genus Mastyyophorus. 
Vol. XXXIV. (continued).

P. 1161. *Bleptina cliruptalis=Megachyta borgesalis, Walk.

P. 1167. Bertula excelsalis belongs to the genus Mastygophorus.

P. 1174. *Gizama anticalis = Renia deceleusalis, Walk.

P. 1180. *Bononia niveilinea $=$ Licha undilinealis, Walk.

P. 1180. *Crymona receptalis $=H$. hastalalis, Walk.

P. 1181. Bolica armata. A distinct species.

P. 1181. *Sorygaza didymata. A distinct species.

P. 1191. Orocala distentalis. Near the genus Notarcha.

P. 1192. *Alicurda vexatilis $=A$. atialis, Walk.

P. 1199. *Tibracana xanthialis. A distinct species.

P. 1224. Maguza albiguttalis=Pastona rudis, Walk.

P. 1225. Pyralis intermedialis = Hypsopygia sodalis, Walk.

P. 1225. * Pyralis externalis = Hedylepta antenoralis, Walk.

P. 1225. *Pyralis varipes =Alicarda atialis, Walk.

P. 1232. Pyralis crassipes is the + of Tosale pyralidoides, Walk.

P. 1262. *Zarania cossalis =Ethnistis munitalis, Led.

P. 1283. *Rhodaria directalis $=$ Hyalorista taniolalis, Gn.

P. 1303. *Samea obliteralis $=$ Sameodes nicausalis, Walk.

P. 1331. Oligostigma amoenalis belongs to the genus Glaphyria.

P. 1331. Oligostigma exhibitalis. A distinct species.

P. 1354. Glyphodes rutilalis $=$ G. suavis, Feld.

P. 1361. Margaronia auricostalis $=$ M. argealis, Walk.

P. 1390. *Botys additalis=Acharana phceopteralis, Gn.

P. 1390. *Botys ineffectalis $=$ Blephoramastix colubralis, Gn.

P. 1400. *Botys fuliginalis = Stenomeles agavealis, Walk.

P. $1400 .{ }^{*}$ Botys cellatalis = Acharana phcoopteralis, Gn.

P. 1466. *Scopula permixtalis belongs to the genus Phlyctcenia.

P. 1492. Bouchis scoparioides belongs to the genus Ethnistis.

P. 1497. *Scoparia stupidalis=Illice batialis, Walk., of the family Lithosiidæ.

Vou. XXXV.

P. 1543. Pyrinia xantharia belongs to the genus Capnodes.

P. 1549. *Azelina imnizundaria $=A$. stuposaria, Gn.

P. 1639. Ceronaba cinctaria = Molybdophora concinnaria, H.-S.

P. 1702. Lephana tetraphorella belougs to the Noctuidæ. Type in B. M.

P. 1743. Gabalceca bilineatella belongs to the genus Erupa. Type in B. M.

P. 1769. Zolca congruella belongs to the genus Erupa. Type in B. M.

P. 1783. Pandemis securiferana. Type in B. M.

P. 1800. Torda metamelana $=$ Tosale pyralidoides, Walk. Type in B. M.

P. 1958. Celona canifimbria belongs to the Noctuidæ. Type in B. M.

P. 1963. Acontia quadrata. Type lost.

P. 1971. Thyridospila caeca. Type lost.

The remaining references are to species described in the Transactions of the Entomological Society for 1862 :-

P. 76. *Cingilia humeralis = Caterva catenaria, $\mathrm{Cr}$.

Proo. Zool. Soo.-1896, No. XLII. 
P. 79. Heterocampa thyatiroides. Not identified.

P. 82. Miresa divergens = Lithacodes fasciola, H.-S.

P. 90. Gora cequalis. Not identified.

P. 94. Erysthia obliquata (locality doubtful). Not identified.

P. 95. Cottobara concinna. A Notodont.

P. 95. Gaphara sobria. Allied to Celana tetera.

P. 97. *Nonia signiplena belongs to the genus Posula.

P. 101. *Asthana erect $a=$ Poesula transversaria, Walk.

P. 101. *Asthana directa belongs to the genus Posula.

P. 102. Baniana incequalis. A distinct species.

P. 103. Gammace magniplaga. Not identified.

P. 104. *Remigia consistens = Renodes brevipalpis, $\mathrm{Gn}_{\mathrm{n}}$.

P. 104. Gangra atripustula belongs to the Thermesiidø.

P. 105. Focilla abrupta belongs to the Thermesiidæ.

P. 108. * Marthama conspersa $=M$. squamivaria, Walk.

P. 109. *Hypena murina $=H$. exoletalis, Gn., in B. M.

P. 115. *Bocana marginalis $=B$. orionalis $\sigma^{\circ}, \mathrm{Walk}$.

P. 122. Salbia varialis belongs to the genus Phostria. In B. M.

P. 122. Asopia depressalis. Not identified.

P. 124. Cataclysta insulalis. A distinct species.

P. 126. Scopula desistalis belongs to the genus Elurlea.

P. 127. Scoparia fascialis= Hapalia illibalis, Hübn.

P. 259. Hadena opima. Not identified.

P. 260. Poaphila plagiata. Not identified.

P. 260. Pocaphila figurata. Not identified.

P. 260. Phurys mensurata. Not identified.

P. 260. *Hypena scissilinea belongs to the genus Saserna and is well figured in the Biol. Centr.-Amer.

P. 271. Rhagonis bicolor. Not identified.

P 277. *Pocilocampa plurilinea=Tolype opalina, Walk.

4. Further Contributions to our Knowledge of the Bornean Lycanida. By Hamilton H. Druce, F.Z.S., F.E.S.

[Received June 15, 1896.]

(Plates XXIX.-XXXI.)

Since June last year, when I had the honour of bringing before the Society a paper ${ }^{1}$ dealing with this family of Butterflies, a large amount of fresh material has come to hand.

Dr. Staudinger has received many more specimens from his collector Waterstradt, and $\mathrm{Mr}$. D. Cator has placed in my hands for examination a number of specimens captured by himself and procured from collectors in the island. Amongst these I have found several of considerable interest, which are now recorded here for the first time. Mr. Cator writes me that the places at which he captured specimens are:-Segalind and Sapagaya, which are rivers falling into different parts of Sandakan Bay; Melikop

1 See P. Z. S. 1895 , p. 556. 


\section{$2 \mathrm{BHL}$ Biodiversity Heritage Library}

Schaus, William. 1896. "3. On Walker's American Types of Lepidoptera in the Oxford University Museum." Proceedings of the Zoological Society of London 1896, 634-650. https://doi.org/10.1111/j.1096-3642.1896.tb03072.x.

View This Item Online: https://www.biodiversitylibrary.org/item/97658

DOI: https://doi.org/10.1111/j.1096-3642.1896.tb03072.x

Permalink: https://www.biodiversitylibrary.org/partpdf/72748

\section{Holding Institution}

Smithsonian Libraries

\section{Sponsored by}

Biodiversity Heritage Library

\section{Copyright \& Reuse}

Copyright Status: Public domain. The BHL considers that this work is no longer under copyright protection.

This document was created from content at the Biodiversity Heritage Library, the world's largest open access digital library for biodiversity literature and archives. Visit BHL at https://www.biodiversitylibrary.org. 